\title{
Blind Equalization With the Lattice Constant Modulus Algorithm
}

\author{
R.A. Axford, Jr. \\ Naval Command, Control and Ocean Surveillance Center \\ RDTE Division, Code 844, San Diego, CA 92152-5733
}

\begin{abstract}
This paper presents an evaluation of the performance of the lattice constant modulus algorithm (LCMA) in blind channel equalization. The convergence performance of LCMA is compared to that of its transversal counterpart in equalizing the distortion of four progressively more stressing finite impulse response channels for 8-PSK and 16-QAM signals. The results indicate that while the convergence behavior of both algorithms depends strongly on the transmitted constellation, LCMA exhibits superior performance for 16QAM as the spectral dynamic range of the channel increases.
\end{abstract}

\section{Introduction}

In traditional adaptive channel equalization schemes, training sequences of known data are periodically sent by the transmitter to allow adjustment of the receiver's equalizer coefficients. Blind equalization techniques accomplish the adjustment of adaptive equalizer coefficients without the use of training sequences. The majority of blind equalization schemes can be categorized as either Bussgang techniques or polyspectra-based techniques [1]. Members of the Bussgang family are typically similar to the LMS algorithm in that stochastic gradient descent techniques are used to minimize a cost function that depends on both the output of the equalizer and some (known or assumed) statistical description of the transmitted signal. The most widely utilized of the Bussgang techniques for blind equalization is the constant modulus algorithm (CMA) [2], [3]. CMA is based on the recognition that multipath distortion and additive interference cause amplitude fluctuations in a signal that, in the absence of these impairments, would otherwise exhibit a constant amplitude (at the slicing instants). CMA is also applicable to signals with multiple amplitudes, such as $M$-ary QAM, in which the "constant modulus" becomes a quantity related to the average amplitude of the constellation. In this paper we will compare transversal and lattice filter implementations of the "2-2" variant of CMA [3], each based on stochastic gradient minimization of the phase-blind cost function

$$
J \triangleq \frac{1}{4} E\left[\left(|\hat{a}(n)|^{2}-R_{2}\right)^{2}\right] ; \quad R_{2} \triangleq \frac{E\left[\left|a_{k}\right|^{4}\right]}{E\left[\left|a_{k}\right|^{2}\right]}
$$

where $\hat{a}(n)$ is the equalizer's estimate of the transmitted symbol, and the expected values in the definition of the constant $R_{2}$ are taken over all symbols $\left\{a_{k}\right\}_{k=1}^{N}$ in the transmitted constellation.

From (1) we note that $J$ depends on the transmitted constellation through the constant $R_{2}$. However, the use of an alternate value, say $\beta R_{2}$, only results in a scaling of the amplitude of $\hat{a}(n)$ by $\beta^{1 / 2}$. While the appropriate selection of $R_{2}$ is important for smooth switching between CMA and the decision-directed mode [4], we see that, except for this scaling, the CMA cost function is independent of the transmitted constellation.

It is well known that CMA converges more slowly and exhibits greater residual mean-square estimation error after convergence for QAM than for PSK signals. These results are not surprising if we consider the error function component of the CMA stochastic gradient weight update:

$$
e(n)=\hat{a}(n)\left(|\hat{a}(n)|^{2}-R_{2}\right) .
$$

For QAM, $e(n)$ is non-zero for perfect symbol estimates while for PSK it is zero for perfect symbol estimates.

It is possible to accelerate blind equalizer convergence for QAM signals by employing variations of CMA that take advantage of knowledge of the transmitted constellation [5], [6]. Here, however, we will study the performance of a CMAconvergence-accelerating technique for which knowledge of the transmitted constellation is unnecessary, namely the lattice constant modulus algorithm (LCMA) [7]. The methods used in this paper are based on the early stochastic gradient lattice (SGL) equalizer work of Satorius and Alexander in [8], and on the more recent comparative evaluation of several blind equalization algorithms by Shynk et al. in [9].

2 The Transversal and Lattice Constant Modulus Algorithms

This section defines the two blind equalization algorithms considered in the paper. In all that follows, use is made of the notational conventions for complex-valued random variables contained in [10].

\subsection{Transversal CMA (TCMA)}

The coefficient update formula of TCMA with a normalized step-size can be expressed as

$$
\mathbf{w}(n+1)=\mathrm{w}(n)-\hat{P}_{\mathrm{u}}^{-1}(n) \hat{a}^{+}(n)\left(|\hat{a}(n)|^{2}-R_{2}\right) \mathrm{u}(n)
$$

where * indicates complex conjugate,

$$
\mathbf{u}(n)=\left[\begin{array}{llll}
u(n) & u(n-1) & \ldots & u(n-L)
\end{array}\right]^{T}
$$

is the vector of samples of the received waveform $u(n)$,

$$
\mathrm{w}(n)=\left[\begin{array}{llll}
w_{0}(n) & w_{1}(n) & \ldots & w_{L}(n)
\end{array}\right]^{T}
$$

is the vector of variable tap-weights at time $n$, 


$$
\hat{a}(n)=\mathbf{w}^{H}(n) \mathbf{u}(n)=\sum_{\ell=0}^{l} w_{\ell}^{*}(n) u(n-\ell)
$$

is the output of the $L+1$ tap equalizer at time $n$,

$$
\hat{P}_{\mathrm{u}}(n)=\left(1-\alpha_{T}\right) \hat{P}_{\mathrm{v}}(n-1)+\mathrm{u}^{H}(n) \mathrm{u}(n)
$$

is a running estimate of the power in the equalizer, and

$$
0<\alpha_{T}<1
$$

is the normalized step-size parameter. The estimate defined in (7) is biased and over-estimates the power in the equalizer by $\alpha_{T}^{-1}$. The appropriate initialization of (7) is

$$
\hat{P}_{v}(0)=(L+1) P_{u} \alpha_{T}^{-1}
$$

where $P_{u}$ is the true power in $u(n)$. Reducing $\alpha_{T}$ decreases both the step size and the asymptotic MSE of the algorithm. Increasing $\alpha_{T}$ allows the algorithm to converge more quickly at the expense of increasing the asymptotic MSE.

\subsection{Lattice CMA (LCMA)}

The structure of the LCMA equalizer is shown in figure 1. The lattice order recursion update equations a re given by

$$
\begin{aligned}
& f_{\ell}(n)=f_{l-1}(n)-K_{\ell}(n) b_{\ell-1}(n-1) \\
& b_{\ell}(n)=b_{\ell-1}(n-1)-K_{\ell}^{*}(n) f_{\ell-1}(n)
\end{aligned}
$$

where $K_{\ell}(n)$ is the $\ell^{\text {th }}$-stage PARCOR coefficient, and $f_{\ell}(n)$ and $b_{\ell}(n)$ are the $\ell^{\ell t}$-order forward and backward predictionerrors, respectively, all at time $n$. Note that $f_{0}(n)=b_{0}(n)=u(n)$. The $\ell^{\text {th }}$-stage PARCOR coefficient is updated according to

$$
K_{\ell}(n+1)=K_{\ell}(n)+\hat{P}_{f b \ell}^{-1}(n)\left[b_{\ell-1}^{*}(n-1) f_{\ell}(n)+f_{\ell-1}(n) b_{\ell}^{*}(n)\right]
$$

where

$$
\hat{P}_{f b l}(n)=\left(1-\alpha_{L}\right) \hat{P}_{f b l}(n-1)+\left[\left|f_{l-1}(n)\right|^{2}+\left|b_{l-1}(n-1)\right|^{2}\right]
$$

is a biased estimate of the total prediction-error power entering the $\ell^{\text {th }}$-stage, and $0<\alpha_{L}<1$ is a normalized step size parameter. The update (12) accomplishes a method-ofsteepest-descent minimization of the cost function

$$
J_{f b \ell} \triangleq \frac{1}{2}\left(E\left[\left|f_{\ell}(n)\right|^{2}\right]+E\left[\left|b_{\ell}(n)\right|^{2}\right]\right) \text {. }
$$

The purpose of the lattice structure in the context of channel equalization is to transform the correlated input sequence $u(n), u(n-1), \ldots, u(n-L)$ into the uncorrelated sequence $b_{0}(n), b_{1}(n), \ldots, b_{L}(n)$ of backward prediction-errors. (It can be shown that the information content of both sequences is identical. See section 4.16 of [10].) The significance of this when training sequences are used can be appreciated by viewing the lattice equalizer as a transversal filter with tapweights (joint process estimation coefficients)

$$
\mathbf{g}(n)=\left[\begin{array}{llll}
g_{0}(n) & g_{1}(n) & \ldots & g_{L}(n)
\end{array}\right]^{T},
$$

"pre-conditioned" input vector

$$
\mathbf{b}(n)=\left[\begin{array}{llll}
b_{0}(n) & b_{1}(n) & \ldots & b_{l}(n)
\end{array}\right]^{r},
$$

and output

$$
\hat{a}(n)=\mathbf{g}^{H}(n) \mathbf{b}(n)=\sum_{\ell=0}^{l} g_{\ell}^{*}(n) b_{\ell}(n)
$$

When training sequences are used and the cost function is simply the mean-squared-error between the equalizer output and the transmitted symbol $a(n)$

$$
J_{M S E} \triangleq E\left[|\hat{a}(n)-a(n)|^{2}\right] \text {, }
$$

the resulting error function component $(e(n)=\hat{a}(n)-a(n))$ of the stochastic gradient weight update is linear. Furthermore, after the PARCOR coefficients have converged, the autocorrelation matrix of $b(n)$

$$
\mathbf{D} \triangleq E\left[\mathbf{b}(n) \mathbf{b}^{H}(n)\right]
$$

is diagonal and given by

$$
\mathbf{D}=\operatorname{diag}\left[E\left[\left|b_{0}(n)\right|^{2}\right] \quad E\left[\left|b_{1}(n)\right|^{2}\right] \ldots E\left[\left|b_{L}(n)\right|^{2}\right]\right] .
$$

Thus the eigenvalues of $D$ consist of the backward predictionerror powers, orders 0 through $L$. Typically, the dynamic range of these powers is less, often much less, than the dynamic range of the eigenvalues of the correlation matrix of the received waveform samples

$$
\mathbf{R} \triangleq E\left[\mathbf{u}(n) \mathbf{u}^{H}(n)\right]
$$

As a result, the quadratic performance surface of the trained stochastic gradient lattice (SGL) equalizer in the space of the tap weights $\mathbf{g}(\mathbf{n})$ will be considerably less distorted than that of the corresponding LMS transversal equalizer in the space of the tap weights $\mathbf{w}(n)$ when the spectral dynamic range of the received signal $u(n)$ is large. The linearity of the "algorithmdriving" error function, combined with the fact that $D$ is diagonal, allows a decoupling of the update equations for the elements of $g(n)$ in the trained SGL equalizer to the extent that

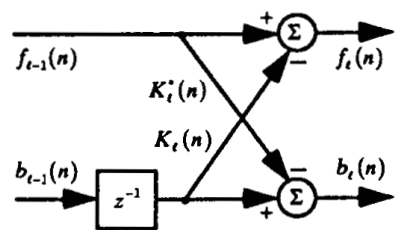

(a)

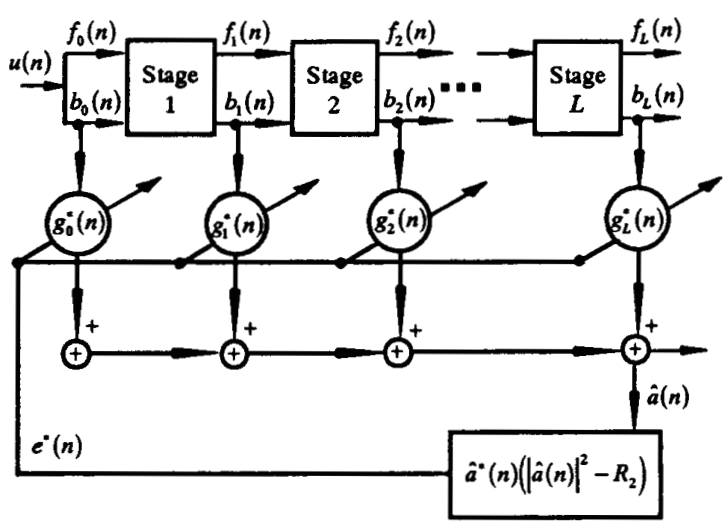

(b)

Figure 1 Lattice CMA Equalizer; (a) single lattice stage; (b) $(L+1)$-tap lattice equalizer using cascaded stages. 
(i) a separate normalized step size, and (ii) an "order-local" error function $v_{\ell}(n)=\hat{a}_{\ell}(n)-a(n)$ may be used in updating each $g_{\ell}(n)[8] .\left(\hat{a}_{\ell}(n)\right.$ is the $\ell^{\text {th }}$-order joint process estimate of $a(n)$.)

In LCMA, since the error function component of the weight update is nonlinear, order-local error terms cannot be used. The update equations for the elements of $\mathbf{g}(n)$ remain coupled through their joint use of $e(n)$ defined in eq. (2). Consequently, some of the "modularity" of the trained SGL equalizer is lost in LCMA. Nonetheless, separate normalized step sizes can still be used for each element of $g(n)$ in LCMA.

The $\ell^{\text {th }}$ variable tap-weight $g_{\ell}(n)$ of the LCMA equalizer is updated according to

$$
g_{\ell}(n+1)=g_{\ell}(n)-\hat{P}_{b \ell}^{-1}(n) \hat{a}^{*}(n)\left(|\hat{a}(n)|^{2}-R_{2}\right) b_{\ell}(n)
$$

where

$$
\hat{P}_{b \ell}(n)=\left(1-\alpha_{L}\right) \hat{P}_{b \ell}(n-1)+\left|b_{\ell}(n)\right|^{2}
$$

is a biased estimate of the $\ell^{\text {th }}$-order backward prediction-error power, and $0<\alpha_{L}<1$ is the normalized step size parameter. Varying $\alpha_{L}$ has the same general effects on the convergence time and asymptotic MSE of LCMA as varying $\alpha_{T}$ does on the performance of TCMA.

\subsection{Blind Equalizer Coefficient Initialization}

In [2], Godard argued that undesirable local minima of the cost function $J$ in the space of the variable tap weights can be avoided by using what has come to be called the "spike initialization". This consists of setting the initial value of all variable tap-weights to zero with the exception of a single weight at or near the center of the equalizer. According to Godard, the initial value of this "reference" tap-weight should be set to a value larger than a constant that depends on moments of the transmitted constellation and "the channel impulse response sample having the largest magnitude" (see eq. (38) of [2]). The universal validity of this assertion has subsequently been disproven [11], [12]. The search for a blind equalizer that will converge to a global minimum regardless of the initial values of the variable tap-weights is a topic that is beyond the scope of this paper. It is sufficient for our purposes to note that while there are situations of significant practical interest for which CMA fails to converge from the "spike initialization", there are also situations of significant practical interest for which CMA does in fact successfully converge using this initialization.

In all simulations that follow, we set $L+1=11$ taps, and the initial tap-weight vectors are always set to

$$
\mathbf{w}(0)=\left[\begin{array}{lllllllllll}
0 & 0 & 0 & 0 & 0 & 1 & 0 & 0 & 0 & 0 & 0
\end{array}\right]^{T}
$$

for TCMA, and

$$
\mathbf{g}(0)=\left[\begin{array}{lllllllllll}
0 & 0 & 0 & 0 & 0 & 1 & 0 & 0 & 0 & 0 & 0
\end{array}\right]^{T},
$$

$$
K_{\ell}(0)=0, \quad \ell=1,2, \ldots, 10
$$

for LCMA. For both algorithms, this corresponds to an initial all-pass-filter equalizer transfer function with a group delay of 5.

\section{Simulation Configuration}

A schematic diagram of the computer simulations is shown in figure 2. The transmitted signal $a(n)$ consists of an uncorrelated sequence of either 8-PSK symbols with unit amplitude, or 16-QAM symbols with real and imaginary components drawn from $\{ \pm 1, \pm 3\}$. The appropriate values of $R_{2}$ from eq. (1) are 1 and 13.2 , respectively.

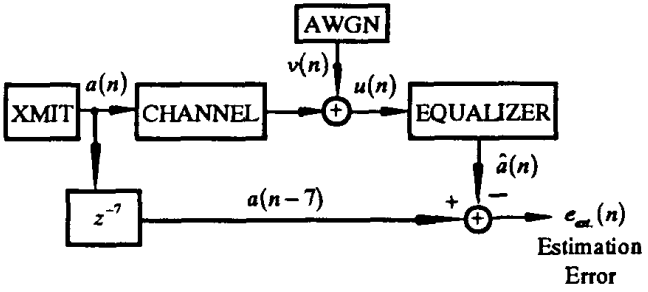

Figure 2 Computer simulation configuration.

The impulse response $h(n)$ of the channel is given by

$$
h(n)=h_{1} \delta(n-1)+h_{2} \delta(n-2)+h_{3} \delta(n-3)
$$

where

$$
h_{k}=\left\{\begin{array}{l}
\frac{1}{2 \sqrt{\mathbf{h}^{T} \mathbf{h}}}\left[1+\cos \left(\frac{2 \pi}{W}(k-2)\right)\right], \quad k=1,2,3 \\
0, \quad \text { otherwise }
\end{array}\right.
$$

and

$$
\mathbf{h}=\left[\begin{array}{lll}
h_{1} & h_{2} & h_{3}
\end{array}\right]^{T}
$$

Thus, the channel's impulse response is normalized such that $h_{1}^{2}+h_{2}^{2}+h_{3}^{2}=1$ for all values of the bandwidth parameter $W$. Except for this normalization, the channel model is the same used by Satorius and Alexander in [8]. Complex additive white Gaussian noise $v(n)$ is added to the output of the channel to form the received waveform $u(n)$. The real and imaginary components of $v(n)$ are independent, zero-mean Gaussian random processes, each with variance 0.001 . The transmitted sequence is delayed by the combined group delay of the channel and equalizer ( 7 samples), and compared to the estimates produced by the equalizer. The estimation error $e_{\text {est. }}(n)$ is formed as shown in figure 2. Given that the channel impulse response samples are real-valued and that perfect carrier recovery is assumed, a decision-directed phase locked loop is not used. Nonetheless, the tap-weights in $w(n)$ and $g(n)$ are rotated at each iteration such that the imaginary component of the reference tap $(\ell=5)$ is kept equal to zero. This was found to be necessary in order to prevent $\hat{a}(n)$ from spinning slightly for the largest values of $\alpha_{T}$

with 


\section{Results}

Figure 3 shows four LCMA learning curves, one corresponding to each value of $W$ as shown, for the 16-QAM signal and $\alpha_{L}=0.0001$. Each curve was obtained by ensemble-averaging $\left|e_{\text {est. }}(n)\right|^{2}$ over 10 independent trials. The ensemble-averaged curves were further smoothed with a 50-point moving-average filter as was done in [9]. Figure 4 shows three LCMA learning curves for $W=2.9$ and the values of $\alpha_{L}$ indicated on the plot. Note that while decreasing $\alpha_{L}$ increases the convergence time, it also reduces the asymptotic MSE $\varepsilon_{\infty}^{2}$.

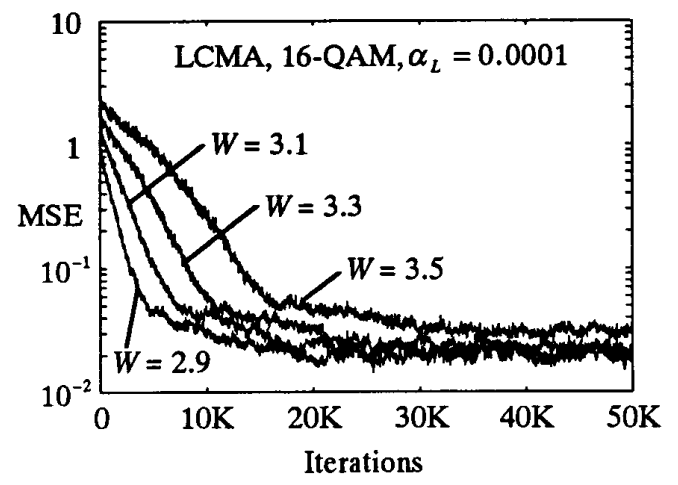

Figure 3 LCMA learning curves for four values of $W$.

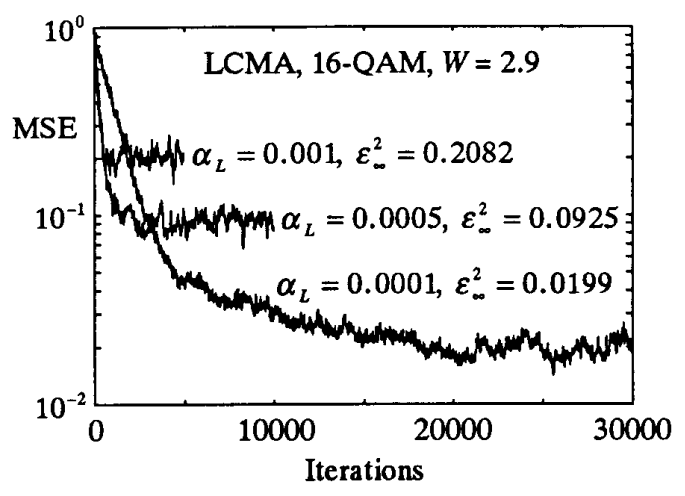

Figure 4 LCMA learning curves for three values of $\alpha_{L}$.

In order to compare the convergence performance of LCMA and TCMA independently of the values of the step-size parameters, several smoothed learning curves were produced for each algorithm using various values of $\alpha_{L}$ and $\alpha_{T}$. From these curves, the asymptotic MSE $\varepsilon_{\infty}^{2}$ and the number of iterations required to reach $\varepsilon_{\infty}^{2}$ were determined and plotted producing convergence performance curves. As noted in [9], when comparing two adaptive equalization algorithms in this way, if one algorithm's performance curves always lie below those of another, then the former algorithm clearly has superior convergence performance.
Figure 5 shows the LCMA (solid) and TCMA (dotted) convergence performance curves for 4 values of $W$ and both modulations. These performance curves summarize the information gathered from a total of 134 smoothed learning curves. The left-hand (right-hand) extreme of any performance curve corresponds to the largest (smallest) step size used. Horizontal dashed lines indicate the MSE levels achieved by an 11-tap Wiener equalizer (cost function defined in eq. (18)) for 8-PSK (unit amplitude) and 16-QAM $(\{ \pm 1, \pm 3\})$ when the thermal noise variance is 0.001 . Note that as $W$ increases, the minimum MSE for this 16-QAM constellation increases more rapidly than does that of the 8-PSK signal. Thus 2 horizontal dashed lines appear for $W \geq 3.3$, the lower of which corresponds to 8-PSK in figures 5 (c) and (d).

For both algorithms, the transmitted constellation is a stronger factor in determining the convergence performance than is the spectral dynamic range of the channel (at least for the constellations and channel parameters used here). Both algorithms always converge more rapidly for 8-PSK than for 16-QAM when channel conditions are equal. Furthermore, both algorithms converge more quickly for 8-PSK under the most stressing channel conditions used in these simulations $(W=3.5)$ than for $16-\mathrm{QAM}$ under the least stressing channel conditions $(W=2.9)$.

For 8-PSK, these simulation results indicate that the convergence performance of LCMA is actually slightly inferior to that of TCMA until the eigenvalue ratio of $\mathbf{R}$ reaches roughly $16 \mathrm{~dB}$. However, for both modulations, LCMA is less sensitive than TCMA to increases in the spectral dynamic range of $u(n)$.

For 16-QAM, the convergence performance of LCMA becomes superior to that of TCMA as the eigenvalue ratio of $\mathbf{R}$ increases above roughly $13 \mathrm{~dB}$. Thus the stochastic gradient lattice can indeed accelerate the convergence of a CMA blind equalizer for amplitude modulated signals.

\section{Summary}

This paper has presented the first evaluation of the convergence performance of the lattice constant modulus algorithm in blind channel equalization. The appropriate "spike initialization" of the LCMA equalizer bas been demonstrated. The clear convergence rate superiority of trained SGL equalizers over trained transversal LMS equalizers is not always observed in the corresponding "2-2" CMA versions of these filters. The convergence performance of both lattice and transversal CMA equalizers depends strongly on the transmitted constellation. However, as the spectral dynamic range of the received signal increases, the convergence performance of LCMA is degraded to a lesser degree than that of TCMA. Further work is required to fully understand effects of the spectral dynamic range of the received signal on the CMA performance surface. 

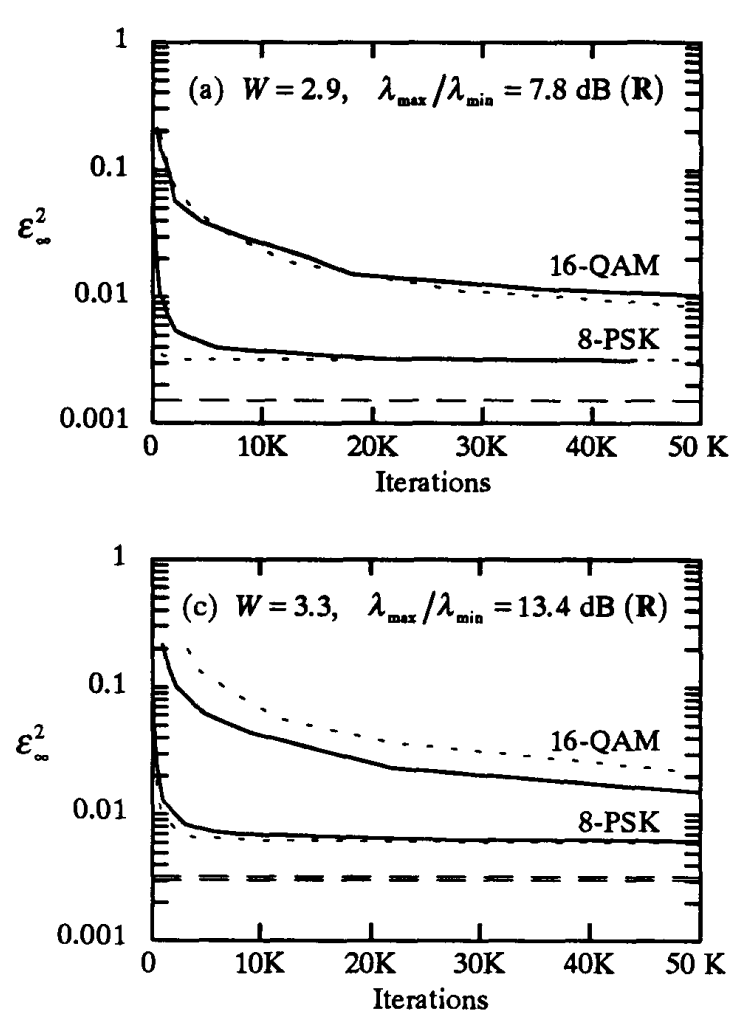
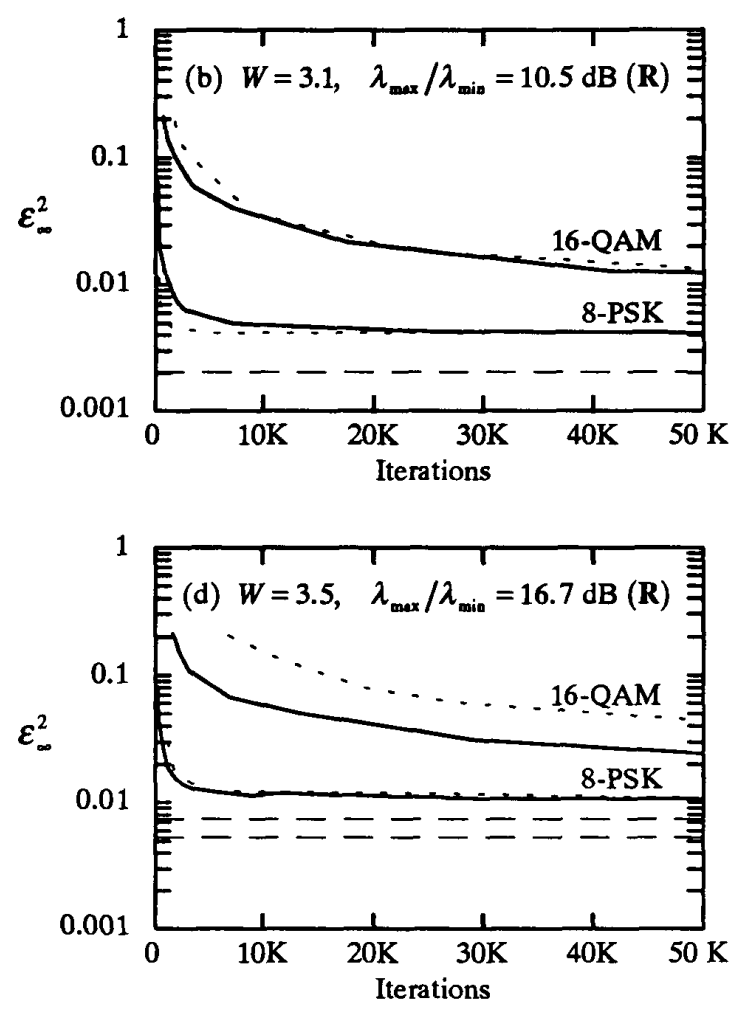

Figure 5 Convergence performance curves for LCMA (solid) and TCMA (dotted). Horizontal dashed lines indicate Wiener MSEs.

\section{References}

[1] J.G. Proakis and C.L. Nikias, "Blind Equalization," (overview paper), in Adaptive Signal Processing, Simon Haykin, Editor, Proc. SPIE 1565, pp. 76-87, San Diego, CA, 22-24 July, 1991.

[2] D.N. Godard, "Self-Recovering Equalization and Carrier Tracking In Two-Dimensional Data Communications Systems," IEEE Trans. on Communications, vol. COM-28, no. 11, pp. 1867-1875, Nov. 1980.

[3] J.R. Treichler and B.G. Agee, "A New Approach to Multipath Correction of Constant Modulus Signals," IEEE Trans. on ASSP, vol. ASSP-31, no. 2, pp. 459-472, April 1983.

[4] N.K. Jablon, "Joint Blind Equalization, Carrier Recovery, and Timing Recovery for Higher-Order QAM Signal Constellations," IEEE Trans. on Signal Processing, vol. 40, no. 6, pp. 1383-1398, June 1992.

[5] M.J. Ready and R.P. Gooch, "Blind Equalization Based on Radius Directed Adaptation," Proc. IEEE ICASSP, pp. 1699 $1702,1990$.

[6] F. Lopez de Victoria, "An Adaptive Blind Equalization Algorithm for QAM and QPR Modulations: The Concentric Ordered Modulus Algorithm, with Results for 16-QAM and 9-
QPR," Proc. 25th Asilomar Conf., pp. 726-730, Pacific Grove, CA, 4-6 Nov., 1991.

[7] R.P. Gooch, M. Ready and J. Svoboda, "A Lattice-Based Constant Modulus Adaptive Filter," Proc. 20th Asilomar Conf., pp. 282-286, Pacific Grove, CA, 10-12 Nov., 1986.

[8] E.H. Satorius, and S.T. Alexander, "Channel Equalization Using Adaptive Lattice Algorithms," IEEE Trans. on Communications, vol. COM-27, pp. 899-905, June 1979.

[9] J.J. Shynk, R.P. Gooch, G. Krishnamurthy and C.K. Chan, "Comparative Performance Study of Several Blind Equalization Algorithms," in Adaptive Signal Processing, Simon Haykin, Editor, Proc. SPIE 1565, pp. 102-117, San Diego, CA, 22-24 July, 1991.

[10] S. Haykin, Adaptive Filter Theory, Prentice Hall, 1986.

[11] Z. Ding, R.A. Kennedy, B.D.O. Anderson and C.R. Johnson, "Ill-Convergence of Godard Blind Equalizers in Data Communications Systems," IEEE Trans, on Communications, vol. 39, no. 9, pp. 1313-1327, September, 1991.

[12] J.R. Treichler, V. Wolff, and C.R. Johnson, "Observed Misconvergence in the Constant Modulus Adaptive Algorithm," Proc. 25th Asilomar Conference, pp. 663-667, Pacific Grove, CA, 4-6 November, 1991. 